\title{
Analytical modeling of liquid sloshing in a two-dimensional rectangular tank with a slat screen
}

\author{
O. M. Faltinsen • R. Firoozkoohi · A. N. Timokha
}

Received: 19 March 2010 / Accepted: 17 July 2010 / Published online: 4 August 2010

(C) The Author(s) 2010. This article is published with open access at Springerlink.com

\begin{abstract}
Potential-flow theory is employed with linear free-surface conditions, multimodal method, and a screenaveraged pressure-drop condition to derive an analytical modal model describing the two-dimensional resonant liquid motions in a rectangular tank with a vertical slat-type screen in the tank middle. The tank is horizontally excited in a frequency range covering the two lowest natural sloshing frequencies. The model consists of a system of linear ordinary differential [modal] equations responsible for liquid sloshing in compartments, as well as a nonlinear ordinary differential equation describing the liquid flow between the compartments. New experimental model tests on steady-state wave elevations near the tank wall are reported for the solidity ratios $0.328 \leq \mathrm{Sn} \leq 0.963$ where $\mathrm{Sn}$ is the ratio between the solid area and the full area of the screen. The experiments generally support the applicability of the model. The discrepancy can be explained by the free-surface nonlinearity. The screen acts as a damping mechanism for low and intermediate solidity ratios, but it causes an increase in the lowest resonant sloshing frequency at higher solidity ratios as if the screen had been replaced by an unperforated wall.
\end{abstract}

Keywords Multimodal method $\cdot$ Perforated screen $\cdot$ Sloshing

\section{Introduction}

Screens and perforated plates can provide important damping of sloshing in a tank. They may also affect the natural sloshing frequencies. An important parameter is the solidity ratio, Sn, which is the ratio of the area of the shadow projected by the screen on a plane parallel to the screen to the total area contained within the frame of the screen. The solidity ratio is between zero and one, where $\mathrm{Sn}=0$ means no screen and $\mathrm{Sn}=1$ means that the screen becomes an unperforated wall.

Perforated plates have been studied in the past century in the context of liquid sloshing in cylindrical fuel tanks of spacecraft (see the NASA report [1, Sect. 4.4]). Other hydrodynamic-screen applications are associated with anti-rolling tanks of ships, tuned liquid dampers (TLD) of tall buildings, swash bulkheads of ships, and perforated plates of oil-gas separators on a floating platform (see for instance [2-5]). A design requirement for anti-rolling tanks and TLDs is that the lowest natural sloshing frequency should not be significantly affected by the screen and

O. M. Faltinsen $(\bowtie) \cdot$ R. Firoozkoohi $\cdot$ A. N. Timokha

Centre for Ships and Ocean Structures and Department of Marine Technology, Norwegian University of Science and Technology,

7491 Trondheim, Norway

e-mail: odd.faltinsen@ntnu.no 
approximately equal to the roll natural frequency and the lowest important structural natural frequency, respectively. The damping should be large. The consequence is that either the wave-induced roll motions of a ship or wind- and earthquake-excited vibrations of tall buildings are clearly reduced. A rough guideline is that the solidity ratio ought to be about 0.5 . The objectives for a swash bulkhead in cargo liquid tanks of ships and perforated plates in oil-gas separators on floating platforms are to provide sloshing damping as well as to change the lowest natural sloshing frequency to a higher frequency range where the wave-induced ship and platform velocities and accelerations are less severe. The consequence is a high solidity ratio.

Our focus is on analytical modeling of liquid sloshing in a rectangular tank with a slat screen (interested readers may find a review on appropriate CFD methods in the recent paper by Maravani and Hamed [5]) to be applicable for $\mathrm{Sn} \geq 0.3$. The excitation is horizontal and harmonic, and the flow is nearly two-dimensional.

Existing analytical modeling techniques typically assume that the screen has a minor effect on the natural sloshing frequencies. Under this assumption, we may use the flow in a clean tank as an ambient flow and estimate the energy dissipation due to the screen. This estimate can be done by empirical drag-force formulations that account for flow parameters such as the Reynolds number and the geometrical screen shape [6, Chap. 6], or employing the empirical pressure-drop condition $[3,4,7,8]$. Thereafter, the found damping rates can be incorporated into either modal $[6,9]$ or equivalent (pendulum, spring-mass etc.) mechanical systems $[3,4]$ by using an equivalent linearization technique in steady-state conditions. Such a procedure based on empirical drag formulation is exemplified in [6, Sect. 6.8] for a screen placed in the middle of a rectangular tank with two-dimensional ambient flow. The ambient flow was described by the linear potential-flow theory of an incompressible liquid. The relatively small effect of viscous boundary-layer damping was incorporated. There was good agreement with the experimental values of damping ratio, steady-state wave amplitude and longitudinal force for a rectangular tank with a wire mesh screen by Warnitschai and Pinkaew [2]. The experimental conditions for forced tank oscillations was a water depth-to-tank length ratio of 0.3, and longitudinal forcing amplitude divided by tank length equal to 0.005. The solidity ratio was 0.48 .

A different procedure has to be followed for higher solidity ratios when the screen significantly affects the resonance sloshing frequencies which tends to the natural frequencies in the compartmental tanks, i.e., the tanks that result from replacing the screen with an unperforated wall. A large change in the pressure and free-surface profiles occurs then across the screen. Which lower solidity ratio causes this change in two-dimensional flow depends during harmonic excitation on the liquid depth-to-the tank width ratio and the ratio between the lateral forcing amplitude and the tank width. When the change in the lowest resonance sloshing frequency occurs, the sloshing is less severe relative to a clearly lower or higher solidity ratio.

The main goal of this paper is to show that employing multimodal methods makes it possible to derive an analytical [modal] model for sloshing in a rectangular tank with a screen which can be applied for any solidity ratio. The corresponding modal system is derived from the original boundary-value problem with linear free-surface conditions by employing the pressure-drop condition as a transmission condition at the screen in a domain decomposition scheme. The pressure-drop condition describes the effect of the jet flow through the screen at higher solidity ratio, and the flow separation (wake) at lower solidity ratios. The modal system explicitly handles discontinuity of the free surface (pressure). New experiments with slat-type screens are reported which support the applicability of the derived modal system when the free-surface nonlinearity does not have a dominant effect.

We start the analysis with the linear sloshing boundary problem formulated for each compartment (Sect. 2.1) and, in addition, introduce two transmission conditions (Sect. 2.2) at the screen. The first transmission condition (Neumann-type) implies continuity of normal velocity, but the second (Dirichlet-type) condition governs the screenaveraged pressure drop. In Sect. 2.2, we discuss applicability of the second condition for the slat screen used in our experiments.

There is a variety of external wave problems with screens and porous barriers that have been studied by using an analytical technique with the corresponding transmission conditions. In the list of these studies, we should mention the papers by E. Tuck $[10,11]$ whose concept of the transmission conditions is, for instance, realized in [12]. Reviews on wave interaction with porous media are given in [13], or in the more recent book [14] where the transmission conditions are based on Darcy's law. Another approach to the pressure-drop condition developed for wind-tunnel engineering is outlined in the review [15] as well as in the handbook [16, pp. 314-316]. It assumes a uniform steady 
flow. Based on this approach, a version of the pressure-drop conditions for external wave problems is adopted in $[17,18]$. The latter papers deal with porous-type screens characterized by small sharp-edged openings providing the flow separation through the holes so that the rotational wakes and jets remain in a neighborhood of the screen. For these screens, a local-averaging procedure is possible (as described by Molin [17]) leading to a pressure-drop condition to be mathematically satisfied at each point of the screen. Our approach averages the pressure-drop condition over the mean submerged screen area by accounting for the exponential decay of the velocity field.

As will be shown in Sect. 2.3, employing the screen-averaged pressure-drop condition leads to non-unique solutions of the linear sloshing problem under consideration. To avoid non-uniqueness, we assume that the velocity profile at the slat screen keeps the same shape for $0 \leq \mathrm{Sn} \leq 1$. In other words, our hypothesis is that the velocity profile (exponentially decaying from the free surface to the bottom) remains close to that occurring for steady-state sloshing in the smooth tank $(\mathrm{Sn}=0)$. This assumption is not in conflict with the asymptotic limit $\mathrm{Sn} \rightarrow 1$. The formula for the pressure loss coefficient $K$ of the screen is taken from [19,20]. Even though the formula is for a steady flow, its applicability for unsteady wave flows was experimentally confirmed in [3].

In Sect. 3, we derive the modal system described above. Because linear free-surface conditions are assumed, the system includes two subsystems of linear ordinary differential equations which governs liquid sloshing in the compartments. This sloshing is excited by both horizontal motions of the tank and cross-flow at the screen. The free-surface elevation described by these subsystems has a jump in the tank middle at the assumed screen position. The presence of the jump is consistent with the fact that there is a pressure drop across the screen. The subsystems are coupled by a nonlinear ordinary differential equation with respect to a generalized coordinate responsible for the liquid flow through the screen.

In Sect. 4, we report new experimental model tests, and compare the experimental measurements on steady-state wave elevations at the tank wall with those following from our modal theory. Agreement is satisfactory and the experiments generally support the theory for smaller forcing amplitude (the forcing amplitude-to-the-tank-width ratio $\eta_{2 a} / l$ is about 0.001$)$. Theoretical predictions for the larger forcing amplitude $\left(\eta_{2 a} / l=0.01\right)$ are consistent in some cases with the primary Fourier harmonics of the measured output signal. The higher harmonics in the measured resonant wave elevations contribute up to $35 \%$ and, we believe (see discussion in [21,22]), they are associated with amplification of the higher modes due to the free-surface nonlinearity. The linear potential-flow theory used here is only relevant in resonant conditions if the damping is sufficiently large. Otherwise nonlinear free-surface effects are dominant [6]. We do not know appropriate analytical studies on nonlinear liquid sloshing in tanks with perforated screens. The proposed analytical modal scheme generally allows for the corresponding generalization.

\section{Statement of the problem}

We consider a two-dimensional rigid rectangular tank with a width $l=2 a$ and a mean liquid depth $h$ (see Fig. 1). A perforated plate (screen) is mounted vertically in the tank middle. The tank is forced horizontally with small amplitudes relative to the tank width. The liquid is incompressible with irrotational flow except in limited jet or wake regions caused by cross-flow at the screen. The free-surface nonlinearity is neglected. Assuming purely piston-like transverse flow velocity at the screen, a steady-state analysis was performed in [6]. The forthcoming study is more

Fig. 1 Geometric definitions for the sloshing analysis with a screen $\mathrm{Sc}_{0}$ in the middle of the tank dividing the tank into two compartments

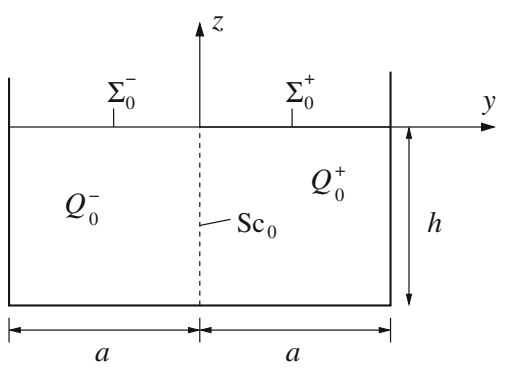




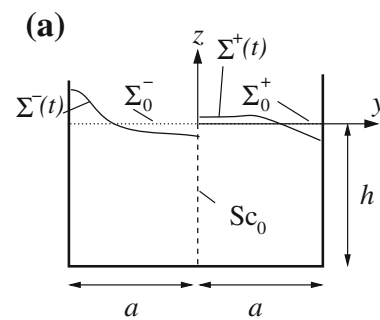
(b)
$\mathrm{Sn}=0$
(c)
$\mathrm{Sn}=1$
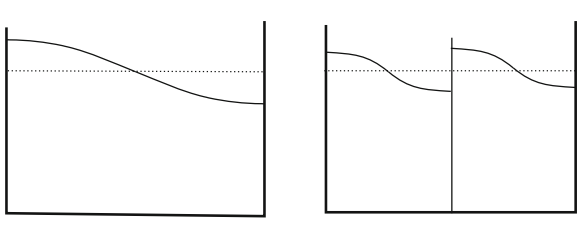

Fig. 2 Typical instantaneous wave profiles for two-dimensional sloshing in a rectangular tank due to horizontal excitation. Screen $\mathrm{Sc}_{0}$ with $0<\mathrm{Sn}<1$ in the middle is installed (part $\mathbf{a}$ ). Parts $\mathbf{b}$ and $\mathbf{c}$ illustrate primary antisymmetric wave profiles for the limit cases $\mathrm{Sn}=0$ and $\mathrm{Sn}=1$, respectively

general. It accounts more properly for the fact that the transverse velocity at the screen decays with depth which matters in particular for larger liquid depths and at higher sloshing frequencies. This latter fact results in a correct prediction of the second natural sloshing frequency, in the present study with $h / l=0.4$ and a frequency range that covers the two lowest natural sloshing frequencies. In addition, the presented modal method may handle transient waves.

\subsection{Boundary-value problem in compartments}

The corresponding linear liquid theory deals with the following boundary-value problems in the unperturbed liquid domains $Q_{0}^{ \pm}$(see Fig. 1).

The theory introduces the absolute velocity potentials $\Phi^{ \pm}(y, z, t)$ in $Q_{0}^{ \pm}$, which should satisfy the Laplace equation and the Neumann boundary conditions on the wetted tank surface

$\nabla^{2} \Phi^{ \pm}=0$ in $Q_{0}^{ \pm} ; \quad \frac{\partial \Phi^{ \pm}}{\partial z}=0(z=-h) ; \quad \frac{\partial \Phi^{ \pm}}{\partial y}= \pm \dot{\eta}_{2}(y= \pm a)$,

where $\eta_{2}(t)$ describes the horizontal motions of the rigid tank.

Furthermore, the theory defines the free-surface elevations $z=\zeta^{ \pm}(y, t)$ and states the kinematic,

$\frac{\partial \Phi^{ \pm}}{\partial z}=\frac{\partial \zeta^{ \pm}}{\partial t} \quad(z=0)$

and dynamic,

$\frac{\partial \Phi^{ \pm}}{\partial t}+g \zeta^{ \pm}=0 \quad(z=0)$

boundary conditions on the unperturbed free surfaces $\Sigma_{0}^{ \pm}$. These conditions couple $\Phi^{ \pm}(y, z, t)$ and $\zeta^{ \pm}(y, t)$ separately for the two compartments. Here, $g$ is the gravity acceleration.

Further, the liquid conservation condition should be fulfilled

$\int_{-a}^{0} \zeta^{-} \mathrm{d} y+\int_{0}^{a} \zeta^{+} \mathrm{d} y=0$.

The problem (1)-(3) requires initial conditions expressing the initial perturbation (function $\zeta_{0}(y)$ ) and its initial velocity (function $\left.\zeta_{1}(y)\right)$ of the free surface to be given, i.e.,

$\zeta(y, 0)=\zeta_{0}(y), \quad \frac{\partial \zeta}{\partial t}(y, t)=\zeta_{1}(y)$.

Even though the free surface is discontinuous at $\mathrm{Sc}_{0}$ (see Fig. 2a), the initial conditions should, due the required time to generate a pressure drop at the screen, involve smooth functions $\zeta_{0}(y)$ and $\zeta_{1}(y)$ on the whole interval $(-a, a)$. That is why the \pm -sign in conditions (5) is omitted. 


\subsection{Transmission conditions on $\mathrm{Sc}_{0}$}

As long as $\mathrm{Sn}=0$ (smooth rectangular tank), the velocity potential and its normal derivative are continuous in the middle provided by an appropriate combination of Neumann and Dirichlet-type boundary conditions (see mathematical details in [23, Chap. 2]). Another limit case, $\mathrm{Sn}=1$, suggests a rigid non-perforated wall in the middle which requires the zero normal derivative; there is no Dirichlet condition stated in this case. For both limit cases (see Fig. 2b, c), there exist analytical [modal] models reducing the linear sloshing problem (1)-(4) to the corresponding linear [modal] systems of linear ordinary differential equations [6, Chap. 5].

As long as the solidity ratio is between 0 and 1 , the first transmission condition does not change, i.e.,

$u_{r}(z, t)+\dot{\eta}_{2}(t)=\frac{\partial \Phi^{-}}{\partial y}=\frac{\partial \Phi^{+}}{\partial y} \quad(y=0)$,

providing equal flux across $\mathrm{Sc}_{0}$. Here $u_{r}(z, t)$ is the relative normal velocity on $\mathrm{Sc}_{0}$.

The second condition should reflect the fact that the hydrodynamic pressure undergoes a jump. This condition is normally formulated for uniform flows which should, generally, be perpendicular to the flat screen. In accordance with definitions in [16] the pressure drop at the screen is

$P^{-}-P^{+}=\frac{1}{2} \rho K U|U|$.

Here, $U=U(t)$ is the horizontal liquid velocity at the screen, $P^{ \pm}=P^{ \pm}(t)$ is the pressure at different sides of the screen so that the right direction is assumed to be positive, $K \geq 0$ is the so-called pressure-loss coefficient depending on the screen geometry, the solidity ratio $\mathrm{Sn}$, the Reynolds number and, generally, the Keulegan-Carpenter number $\mathrm{KC}$ which may be defined as $\mathrm{KC}=U_{M} T / D$ where $U_{M}$ is the amplitude of $U, T$ is the forced oscillation period and $D$ is a characteristic length of the screen such as the slat height. When the uniform in-flow velocity is not perpendicular to the flat screen, the pressure-loss coefficient becomes also a function of the in-flow angle.

When discussing the condition (7), we should remark that all the forces due to viscous effects are commonly expressed as quadratic functions of a reference velocity. An example for that is the Morison's equation commonly used for offshore structures. Formulations such as these rely on experimental values for drag coefficients. The formulations must give a good fit to experimental data. Then comes what parameters affect the drag coefficient. As we have mentioned, that is, for instance, the Reynolds number, the Keulegan-Carpenter number, the structural form (see [21, Chap. 7] where this is extensively discussed). Here we talk about a pressure-loss coefficient which has a similarity with the drag formulation in Morison's equation when viscous-flow separation matters. It is once more an empirical formulation that has been extensively investigated for ambient steady and space-independent flow [16]. Using linear formulations will not fit experimental data.

Our focus is on the slat screens depicted in Fig. 3. They are characterized by openings with sharp edges where flow separation occurs. The latter fact implies that the Reynolds-number dependence of the pressure-loss coefficient is negligible. The values of $K$ for the slat screens with $\mathrm{Sn}>0.3$ and $\mathrm{KC}>2$ (with characteristic dimension equal to the slat height) are, according to [3] (the authors refer to [19,20] where the following expressions were established for steady flow), approximated by the formulas

$K=\left(\frac{1}{\mathrm{Cc}(1-\mathrm{Sn})}-1\right)^{2}, \quad \mathrm{Cc}=0.405 \exp (-\pi \mathrm{Sn})+0.595$.

The pressure-loss coefficient monotonically increases with the solidity ratio from zero (at $\mathrm{Sn}=0$ ) to infinity (at $\mathrm{Sn}=1)$.

Even though formulas (8) are originally proposed for steady uniform flows, it was illustrated in [3] by experiments for certain values of the solidity ratios that (8) is applicable in a screen-averaged sense (with mean values of the velocity and pressure over $\mathrm{Sc}_{0}$ ) for sloshing problems with vertical screens, namely, it holds

$\frac{1}{h} \int_{-h}^{0}\left[\frac{\partial \Phi^{+}}{\partial t}-\frac{\partial \Phi^{+}}{\partial t}\right] \mathrm{d} z=\frac{1}{2} K U|U|, \quad$ where $U=\frac{1}{h} \int_{-h}^{0} u_{r} \mathrm{~d} z$. 
Fig. 3 A schematic distribution of the openings for different value of solidity ratios in our experimental slat screen. The dimensions are in meters. The screen thickness is $5 \mathrm{~mm}$; it is neglected in our analysis. Because there is a limited number of slats, the solidity ratio varies with the mean liquid depth depending on how many openings become submerged. For the liquid depth $0.4 \mathrm{~m}$, the solidity ratio is (from left to right) $0.328,0.52,0.713,0.808$, $0.855,0.905,0.928,0.948$ and 0.963
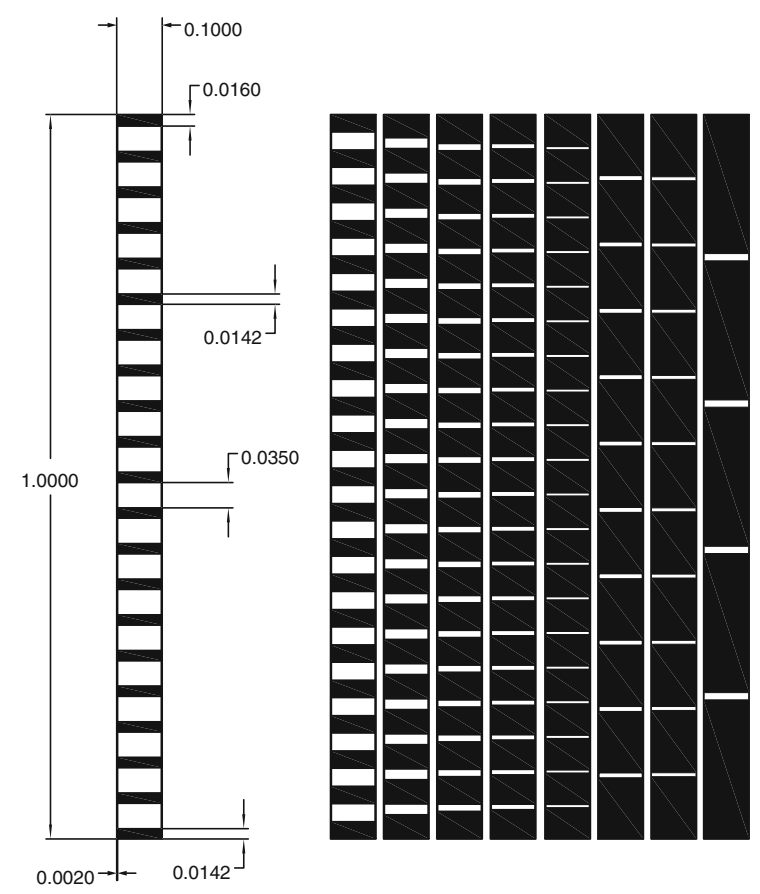

This condition will be adopted in our forthcoming analysis.

The pressure-drop condition plays the role of damping at small and intermediate solidity ratios. When Sn tends to 1 , the flow experiences the screen as a barrier that is difficult to flow through. The consequences are small damping due to cross-flow and that the flow as a first approximation behaves as if the screen is an unperforated wall. The result is that the lowest resonant frequency changes to that in the separated compartments. The change of the resonant frequencies depends on the forcing amplitude, i.e., it cannot be explained by linear theory. Both theory and experiments confirm this fact.

\subsection{Non-uniqueness and additional assumptions}

According to the multimodal method, the velocity potentials $\Phi^{ \pm}$should be presented by a Fourier-type series in the natural sloshing modes (see [6, Chap. 5]). For the whole tank without screen, there are antisymmetric (relative to the $O z$-axis) and symmetric natural modes. The antisymmetric (odd) modes take the form

$\varphi_{i}(y, z)=\sin \left(\frac{1}{2} \pi(2 i-1) y / a\right) \frac{\cosh \left(\frac{1}{2} \pi(2 i-1)(z+h) / a\right)}{\cosh \left(\frac{1}{2} \pi(2 i-1) h / a\right)}, \quad i=1,2, \ldots$

Only these modes are directly excited within the framework of the linear sloshing approximation as $\mathrm{Sn}=0$.

The symmetric (even) natural modes are

$\phi_{i}(y, z)=\underbrace{\cos (\pi i y / a)}_{f_{i}(y)} \frac{\cosh (\pi i(z+h) / a)}{\cosh (\pi i h / a)}, \quad i=1,2, \ldots$

These modes are not excited (within the framework of linear sloshing theory) for $\mathrm{Sn}=0$, but, whereas $\mathrm{Sn}=1$, projections of these modes,

$\phi_{i}^{ \pm}=\left.\phi_{i}\right|_{Q_{0}^{ \pm}}, \quad f_{i}^{ \pm}=\left.f_{i}\right|_{\Sigma_{0}^{ \pm}}$,

constitute the full set of the natural sloshing modes for sloshing in the compartment tanks followed by replacing the screen with a rigid non-perforated wall in the middle. This means that the natural sloshing modes $\phi_{2 n-1}^{ \pm}$are directly excited by lateral tank motions for the limit case $\mathrm{Sn}=1$ (see the wave profile associated with $\phi_{1}^{ \pm}$in Fig. 2c). 
The natural modes $\varphi_{i}$ and $\phi_{i}$ correspond to the natural sloshing frequencies $\sigma_{2 i-1}$ and $\sigma_{2 i}$, respectively, where $\sigma_{k}^{2}=g \kappa_{k}, \quad \kappa_{k}=\frac{\pi k}{2 a} \tanh \left(\frac{\pi k h}{2 a}\right), \quad k \geq 1$.

The antisymmetric modes $\varphi_{i}$ determine the relative horizontal velocity in the tank middle, but give zero contribution to the hydrodynamic pressure at $\mathrm{Sc}_{0}$. Contrary, the symmetric modes $\phi_{i}$ do not affect the relative horizontal velocity at $y=0$, but determine the linear hydrodynamic pressure (Dirichlet trace of the velocity potential) in the tank middle. Because the transmission conditions (6) and (9) imply continuous horizontal velocity, but discontinuous pressure, the absolute velocity potentials $\Phi^{ \pm}$take the form

$\Phi^{ \pm}(y, z, t)=\dot{\eta}_{2} y+\sum_{i=1}^{\infty} \dot{V}_{i}(t) \varphi_{i}(y, z)+\sum_{i=0}^{\infty} R_{i}^{ \pm}(t) \phi_{i}^{ \pm}(y, z)$ in $Q_{0}^{ \pm}$,

where $V_{i}$ and $R_{i}^{ \pm}$are originally unknown time-dependent functions (generalized coordinates). Here, $\dot{\eta}_{2} y$ expresses the liquid motions as a solid body. Modal solution (13) automatically satisfies (1) and (6).

The relative horizontal velocity $u_{r}$ at $y=0$ is

$u_{r}(z, t)=\frac{\pi}{2 a} \sum_{i=1}^{\infty} \dot{V}_{i}(t) \frac{(2 i-1) \cosh \left(\frac{1}{2} \pi(2 i-1)(z+h) / a\right)}{\cosh \left(\frac{1}{2} \pi(2 i-1) h / a\right)}$.

Because the $\left\{\cosh \left(\lambda_{i} y\right), \quad \lambda_{0}<\lambda_{1}<\cdots\right\}$-type exponential functions constitute a complete set of functions on the interval $[0, a]$ for any positive $a$ (see [24, Chap. 1]), any function $u_{r}(z, t)$ can uniquely be presented in the series (14) on the interval $[-h, 0]$. Let us fix $\left\{V_{i}(t), i \geq 1\right\}$ and find $\zeta^{ \pm}(y, t)$ and $R_{m}^{ \pm}(t)$ by solving (2)-(4). Appropriate solution is possible by using, for instance, a Fourier technique from [6, Sect. 5.4.2.4] developed for the problem on liquid sloshing due to deformations of the side wall governed by the normal velocity $u_{r}(z, t)$. Due to this solution, $\zeta^{ \pm}(y, t)$ and $R_{m}^{ \pm}(t)$ become linearly dependent functions of the generalized coordinates $\left\{V_{i}(t), i \geq 1\right\}$. Furthermore, substituting $R_{m}^{ \pm}(t)$ in the modal representation (10) and the integral-type transmission condition (9), we arrive at a single scalar relation coupling the infinite set of functions $\left\{V_{i}\right\}$. This single relation for the infinite set of input generalized coordinates can not provide a unique solution.

To get a unique solution from this single relation, $V_{i}(t)$ must be functions dependent on a single generalized coordinate $\beta_{-1}(t)$. Appropriate $\beta_{-1}(t)$ may be obtained from the assumption that the velocity profile at $\operatorname{Sc}_{0}$ (but not the amplitude!) weakly depends on $K$ (or Sn), namely, remains close to that occurring for the steady-state solution in the limit case $K=0$. Adopting this velocity profile poses no conflict with another limit case $K \rightarrow \infty(\mathrm{Sn}=1)$. Passage to $\mathrm{Sn}=1$ implies that the amplitude parameter $\beta_{-1}(t)$ tends to zero.

Faltinsen and Timokha [6, Sect. 5.4] give the steady-state solution for $\mathrm{Sn}=0$ (smooth rectangular tank) occurring due to harmonic forcing. The corresponding relative velocity potential $\varphi=\Phi-\dot{\eta}_{2} y$ is then as follows

$$
\varphi(y, z, t ; \sigma)=\underbrace{\left(-\sigma \eta_{2 a} \sin (\sigma t) \frac{8 N_{r}}{\pi^{2}}\right)}_{\mathrm{f}(t)} \underbrace{\sum_{i=1}^{\infty} \frac{a(-1)^{i} \sin \left(\frac{1}{2} \pi(2 i-1) y / a\right)}{N_{r}(2 i-1)^{2}\left(1-\bar{\sigma}_{2 i-1}^{2}\right)} \frac{\cosh \left(\frac{1}{2} \pi(2 i-1)(z+h) / a\right)}{\cosh \left(\frac{1}{2} \pi(2 i-1) h / a\right)}}_{\varphi_{-1}(y, z ; \sigma)} .
$$

Here, $\eta_{2}(t)=\eta_{2 a} \cos (\sigma t)$, where $\sigma$ is the forcing frequency, and $\eta_{2 a}$ is the forced sway amplitude of the tank; $\bar{\sigma}_{i}=\sigma_{i} / \sigma$, and

$$
N_{r}=\sqrt{\sum_{i=1}^{\infty} \frac{1}{(2 i-1)^{4}\left(1-\bar{\sigma}_{2 i-1}^{2}\right)^{2}}}
$$

is the norm providing the finiteness of $\varphi_{-1}$ for any forcing frequency $\sigma$. The function $\varphi_{-1}$ in (15) depends on the forcing frequency $\sigma$ and determines the relative horizontal velocity at $y=0$ expressed in terms of $\mathrm{f}(t)$ and $\varphi_{-1}$

$$
u_{r}(z, t ; \sigma)=\left.\mathrm{f}(t) \frac{\partial \varphi_{-1}}{\partial y}\right|_{y=0}=\mathrm{f}(t) \underbrace{\sum_{i=1}^{\infty} \frac{\pi(-1)^{i}}{2 N_{r}(2 i-1)\left(1-\bar{\sigma}_{2 i-1}^{2}\right)} \frac{\cosh \left(\frac{1}{2} \pi(2 i-1)(z+h) / a\right)}{\cosh \left(\frac{1}{2} \pi(2 i-1) h / a\right)}}_{U_{r}(z ; \sigma)} .
$$


Equation (16) defines $U_{r}(z)$ which represents the horizontal velocity profile along the interval $[-h, 0]$ for the considered steady-state sloshing with $\mathrm{Sn}=0$, namely, without screen. The time-dependent function $\mathrm{f}(t)$ is then an amplitude parameter defined by (15). As we discussed above, we impose the horizontal velocity profile $U_{r}$ to keep the same shape for $0<\mathrm{Sn}<1$, i.e., when there is a screen. The screen effect is therefore associated with a modification of the time-dependent function $\mathrm{f}(t)$. We replace the harmonic function $\mathrm{f}(t)$ by an unknown generalized coordinate $\dot{\beta}_{-1}(t)$ governing the magnitude of the profile $U_{r}(z)$ when Sn is not zero. Comparing (14) and (16) leads to the conclusion

$\dot{V}_{i}=\dot{\beta}_{-1}(t) \frac{a(-1)^{i}}{N_{r}(2 i-1)^{2}\left(1-\bar{\sigma}_{2 i}^{2}\right)}$.

The assumption (17) makes the problem (1)-(4), (6), (9) uniquely solvable with the corresponding initial conditions (5). The velocity potential (13) then takes the form

$\Phi^{ \pm}(y, z, t)=\dot{\eta}_{2} y+\dot{\beta}_{-1}(t) \varphi_{-1}(y, z)+\sum_{i=0}^{\infty} R_{i}^{ \pm}(t) \phi_{i}^{ \pm}(y, z)$ in $Q_{0}^{ \pm}$.

\section{Analytical modal model}

According to the multimodal methods, the free surfaces $\Sigma^{ \pm}(t)$ (see Fig. 2) are described (in the tank-fixed coordinate system $O y z$ ) by the Fourier-type representation

$z=\zeta^{ \pm}(y, t)=\beta_{-1}(t) f_{-1}(y)+\sum_{i=1}^{\infty} \beta_{i}^{ \pm}(t) f_{i}(y)$,

where $f_{-1}(y)=\partial \varphi_{-1} / \partial z$ at $z=0$ and $\beta_{-1}(t)$ is the same as in (18).

The component $\beta_{-1}(t) f_{-1}(y)$ is continuous on $(-a, a)$ for any instant $t$. It is responsible for the wave elevations due to liquid flow between the compartments, the time-dependent mean liquid level in the compartments, and provides the volume-conservation condition with

$\int_{-a}^{0} f_{-1}(y) \mathrm{d} y=-\int_{0}^{a} f_{-1}(y) \mathrm{d} y \neq 0$.

Projections of $f_{i}(y), i \geq 1$ on $(-a, 0)$ and $(0, a)$ are exactly the natural surface modes on $\Sigma_{0}^{-}$and $\Sigma_{0}^{+}$, respectively. These satisfy volume conservation in the compartments, namely, $\int_{-a}^{0} f_{i} \mathrm{~d} y=\int_{0}^{a} f_{i} \mathrm{~d} y=0, i \geq 1$.

Substituting (19) and (18) in relations (2) and (3) and using orthogonality of $\left\{f_{n}(y), n \geq 0\right\}$ on the intervals $[-a, 0]$ and $[0, a]$ gives

$\dot{R}_{0}^{ \pm}= \pm\left[-\frac{1}{2} \ddot{\eta}_{2}+v_{0}^{*} \ddot{\beta}_{-1}+g v_{0} \beta_{-1}\right], \quad R_{n}^{ \pm}=\frac{\dot{\beta}_{n}^{ \pm}}{\kappa_{2 n}}$

and the following system of linear modal equations

$\ddot{\beta}_{n}^{ \pm}+\sigma_{2 n}^{2} \beta_{n}^{ \pm}= \pm 2 \kappa_{2 n}\left(a_{n} \ddot{\eta}_{2}+v_{n}^{*} \ddot{\beta}_{-1}+g v_{n} \beta_{-1}\right), \quad n \geq 1$,

where

$$
\begin{aligned}
& a_{n}=\int_{-a}^{0} y f_{n} \mathrm{~d} y=-\frac{(-1)^{n}-1}{\pi^{2} n^{2}} a, \\
& v_{n}^{*}=\int_{-a}^{0} \varphi_{-1} f_{n} \mathrm{~d} y=\frac{2 a}{\pi N_{r}} \sum_{i=1}^{\infty} \frac{(-1)^{i+1}}{\left((2 i-1)^{2}-4 m^{2}\right)(2 i-1)\left(1-\bar{\sigma}_{2 i-1}^{2}\right)}, \\
& v_{n}=\left.\int_{-a}^{0} \frac{\partial \varphi_{-1}}{\partial z}\right|_{z=0} f_{n} \mathrm{~d} y=\frac{1}{N_{r}} \sum_{i=1}^{\infty} \frac{(-1)^{i+1} \tanh \left(\frac{1}{2} \pi(2 i-1) h / a\right)}{\left((2 i-1)^{2}-4 m^{2}\right)\left(1-\bar{\sigma}_{2 i-1}^{2}\right)} .
\end{aligned}
$$


The modal system (21) governs the generalized coordinates determining the component $\sum_{i=1}^{\infty} \beta_{i}^{ \pm}(t) f_{i}(y)$ in representation (19). This component satisfies the volume-conservation conditions on $\Sigma_{0}^{-}$and $\Sigma_{0}^{+}$, and, therefore, does not describe the time-dependent change of the mean liquid levels in the compartments. It looks similar to the case when the compartments are separated by a rigid non-perforated wall. However, in our case, $\beta_{n}^{ \pm}$also depend on the inflow/outflow through $\mathrm{Sc}_{0}$ associated with the generalized coordinate $\beta_{-1}(t)$. Formulas (20) give $R_{n}^{ \pm}(t), n \geq 0$ in Eq. (18) as functions of $\beta_{n}^{ \pm}(t)$ and $\beta_{-1}(t)$.

Furthermore, the right-hand sides in (21) are proportional to

$$
a_{n} \ddot{\eta}_{2}+v_{n}^{*} \ddot{\beta}_{-1}+g v_{n} \beta_{-1}=\int_{\Sigma_{0}^{-}}\left(y \ddot{\eta}_{2}+\varphi_{-1} \ddot{\beta}_{-1}+g \frac{\partial \varphi_{-1}}{\partial z} \beta_{-1}\right) f_{n} \mathrm{~d} S .
$$

When $\dot{\beta}_{-1}(t)$ coincides with $\mathrm{f}(t)$, i.e., when there is no screen for a horizontal harmonic forcing, expression (23) vanishes. As a consequence, the right-hand sides in modal equations (20) becomes zero. This means that we can expect amplification of $\beta_{n}^{ \pm}, n \geq 1$ only for non-small solidity ratios.

Using formulas (20) in representation (18) and substituting them in the transmission condition (9) gives the following ordinary nonlinear differential equation with respect to $\beta_{-1}(t)$

$2 h v_{0}^{*} \ddot{\beta}_{-1}-\left[\frac{K}{2 h} \int_{-h}^{0} U_{r}(z) \mathrm{d} z\left|\int_{-h}^{0} U_{r}(z) \mathrm{d} z\right|\right] \dot{\beta}_{-1}\left|\dot{\beta}_{-1}\right|+2 g h v_{0} \beta_{-1}-a h \ddot{\eta}_{2}+a^{2} \sum_{n=1}^{\infty} \frac{\ddot{\beta}_{n}^{+}-\ddot{\beta}_{n}^{-}}{(\pi n)^{2}}=0$.

An equivalent form of (24) can be written as follows

$$
\left[a(\sigma) \ddot{\beta}_{-1}+b(\sigma) \dot{\beta}_{-1}(t)\left|\dot{\beta}_{-1}(t)\right|+c(\sigma) \beta_{-1}+d(\sigma) \ddot{\eta}_{2}\right]+g a \sum_{n=1}^{\infty} \frac{\tanh (\pi n h / a)}{\pi n}\left(\beta_{n}^{-}-\beta_{n}^{+}\right)=0,
$$

where $\beta_{n}^{ \pm}(t)$ are solutions of (21) and

$$
\begin{aligned}
a(\sigma)= & \left.\int_{-a}^{0} \varphi_{-1}\right|_{z=0} \mathcal{F}(y) \mathrm{d} y=2 h v_{0}^{*}+4 a \sum_{n=1}^{\infty} \frac{\tanh (\pi n h)}{\pi n} v_{n}^{*}, \\
b(\sigma)= & -\frac{K}{2 h} \int_{-h}^{0} U_{r}(z) \mathrm{d} z\left|\int_{-h}^{0} U_{r}(z) \mathrm{d} z\right|, \\
c(\sigma)= & \left.\int_{-a}^{0} \frac{\partial \varphi-1}{\partial z}\right|_{z=0} \mathcal{F}(y) \mathrm{d} y=g\left[2 h v_{0}+4 a \sum_{n=1}^{\infty} \frac{\tanh (\pi n h)}{\pi n} v_{n}\right], \\
d(\sigma)= & \int_{-a}^{0} y \mathcal{F}(y) \mathrm{d} y=-a h+8 a \sum_{i=1}^{\infty} \frac{\tanh (\pi(2 i-1) h)}{\pi^{3}(2 i-1)^{3}}
\end{aligned}
$$

with

$$
\mathcal{F}(y)=2 h+4 a \sum_{n=1}^{\infty} \frac{\tanh (\pi n h)}{\pi n} \cos (\pi n y) .
$$

The system of differential equations (21), (25) couples the generalized coordinates $\beta_{-1}(t), \beta_{n}^{ \pm}(t), n \geq 1$. It can be solved by any numerical method subject to the initial conditions $\beta_{-1}(0)=\beta_{-1}^{(0)}, \beta_{n}(0)=\beta_{n}^{(0)}, n \geq 1$, and $\dot{\beta}_{-1}(0)=\beta_{-1}^{(1)}, \dot{\beta}_{n}(0)=\beta_{n}^{(1)}, n \geq 1$. In our calculations, we used the fourth-order Runge-Kutta method. These initial conditions govern the initial free-surface shape and initial free-surface velocity defined by (5) and (19). 


\section{Steady-state sloshing due to harmonic horizontal excitation}

\subsection{Preliminaries}

We consider steady-state sloshing due to harmonic sway excitation $\eta_{2}(t)=\eta_{2 a} \cos (\sigma t)$, where $\eta_{2 a}$ is the forcing amplitude, and the forcing frequency $\sigma$ is in a range including the two lowest natural sloshing frequencies, $\sigma_{1}$ and $\sigma_{2}$. The steady-state sloshing corresponds to the $2 \pi / \sigma$-periodic solution of the system (21), (25).

As before, there are two limit cases associated with sloshing in a smooth tank (there is no screen, $K=0$ ) and sloshing in two smooth compartments without cross-flow (the screen is an unperforated wall, $K=\infty, \mathrm{Sn}=1$ ). Straightforward (but tedious) algebra shows that, if $K=0$ ( $\mathrm{Sn}=0)$, the steady-state (periodic) solution of (21), (25) is $\dot{\beta}_{-1}=\mathrm{f}(t)$ and $\beta_{n}^{ \pm}(t)=0, n \geq 1$. This solution implies the resonant behavior at $\sigma_{1}$, but not at $\sigma_{2}$, because the symmetric modes are not excited within the framework of linear sloshing theory.

Passage to $K=\infty(\mathrm{Sn}=1)$ in (25) leads to $\dot{\beta}_{-1}(t)=0$ which gives together with the fact that both compartments, by definition, contain the same liquid volumes, that $\beta_{-1}(t)=0$. Substituting $\beta_{-1}(t)=0$ in modal equations (21) gives the well-known linear modal equations for sloshing in smooth rectangular tanks (compartments) with the width $a$ and the filling liquid depth $h$; see [6, Chap. 5]. For this case, the limit $\sigma \rightarrow \sigma_{2}$ leads to the linear resonance response by modes $\phi_{1}^{ \pm}=\left.\phi_{1}\right|_{Q_{0}^{ \pm}}$with wave profiles shown in Fig. 2c, but there is no resonance at $\sigma=\sigma_{1}$.

\subsection{Model tests}

A rectangular tank was installed in a rig located at the Marine Technology Center in Trondheim, Norway. The Plexiglas-made tank dimensions are $1.0 \mathrm{~m} \times 0.98 \mathrm{~m} \times 0.1 \mathrm{~m}$ (width $\times$ height $\times$ breadth). The tank was forced horizontally with a sinusoidal signal. To provide a nearly two-dimensional sloshing, a slat screen (Fig. 3) was used. Meniscus effects at the intersection between the free surface and the tank walls are secondary and cause three-dimensional capillary waves that are riding on the gravity waves.

The tank was equipped with two resistant wave probes installed $1 \mathrm{~cm}$ away from the end walls in order to avoid possible local run-up effects which cannot be described by this theory. The wave elevation is recorded with a sampling rate of $100 \mathrm{~Hz}$ and measured relative to the unperturbed free surface. The error in the measured wave elevation is less than $1 \mathrm{~mm}$.

Our focus is on model tests with $h / l=0.4$ and nondimensional forcing amplitudes close to $\eta_{2 a} / l=0.001$ and 0.01 . The frequency range covers the two lowest natural sloshing frequencies. The wide range of tested solidity ratios of the submerged screen part, as it is accepted in our two-dimensional sloshing analysis with $h / l=0.4$, is listed in the caption of Fig. 3. Note that the actual experimental setup included narrow (about $5 \mathrm{~mm}$ ) vertical rigid constraints mounting the screen to the tank walls. The effects of these constraints on perturbing a local three-dimensional flow at the mounting lines, as well as on the solidity ratio of the considered screens as three-dimensional structures, are neglected. The fact that the slot height is not perfectly constant across the screen is an error source for high-solidity ratios.

To account for the free-surface nonlinearity hysteresis effect (see [6, Chap. 8]), the forcing frequency for a prescribed forcing amplitude changes 'stepwise' after reaching an experimental steady-state regime. The signal with a fixed forcing frequency lasts for about 300 cycles; 200 cycles were sufficient to reach the steady-state condition. After 300 cycles, the forcing frequency changes to a lower value. The reason for decreasing the forcing frequency is to detect the maximum wave elevation. The latter fact follows from the character of the nonlinear steady-state response at a depth larger than the critical depth for sloshing in a smooth two-dimensional rectangular tank (see [6, Chap. 8]). The initial runs were performed with frequency steps of about $0.05 \mathrm{~Hz}$ which enabled localization of the peaks in the steady-state wave-amplitude response. Afterwards, the frequency steps were in the range of $0.01 \mathrm{~Hz}$ to $0.001 \mathrm{~Hz}$, in the frequency domains where the initial tests have detected the resonance peaks in order to more accurately quantify the response peaks. The forcing amplitude might slightly change for technical reasons when switching to another forcing frequency. 


\subsection{Comparison with experiments}

Numerical steady-state solutions are found by using direct numerical simulations by the modal system (21), (25). Our simulations adopt the experimental stepwise change of the forcing frequency and corresponding amplitude with the consequence that simulations with each new forcing parameters employed the initial conditions from the previous steady-state solution. Because (21) and (25) imply a dissipative mechanical system, the long-time simulations always led to a periodic (steady-state) numerical solution. Usually, about 300 cycles are required to get this solution within a two-digits accuracy. This is generally consistent with experiments. The final simulations include up to 1500 cycles providing a four-five digits accuracy for the periodic solution.

When $h / l=0.4$, the lowest theoretical resonances for $\mathrm{Sn}=0$ and $\mathrm{Sn}=1$ are at $\sigma / \sigma_{1}=1$ and $\sigma / \sigma_{1}=\sigma_{2} / \sigma_{1} \approx$ 1.52 with $\sigma_{1}=5.12(\mathrm{rad} / \mathrm{s})$, respectively.

Comparison of theoretical and experimental maximum steady-state wave elevation as a function of forcing frequency is presented in Figs. 4, 5, 6, 7, and 8. The figures show the theoretical values (solid lines), the actual measured maximum wave elevations (empty boxes) at the wave probe (in left compartment), and the amplitude of the primary harmonics of the experimental output signal (associated with $\exp (\mathrm{i} \sigma t), \mathrm{i}^{2}=-1$ ) marked by the filled boxes. The figures give also information on the experimental forcing amplitude as a function of the forcing frequency.

The experiments showed in some cases that different steady-state solutions occur in the compartments in a neighborhood of $\sigma / \sigma_{1}=\sigma_{2} / \sigma_{1}=1.52$. The presence of two stable steady-state solutions is a well-known fact for sloshing in a two-dimensional rigid rectangular tank (here, the compartments) with a finite liquid depth [21,22]. The reason is the free-surface nonlinearity which is not included in the present theory. The corresponding experimental records with different steady-state results in the two compartments are therefore not included in the present comparison.

\subsubsection{Lower values of the solidity ratio}

Figure 4 shows experimental and numerical values of the maximum wave elevations for $\mathrm{Sn}=0.328$ and 0.52 ( $K=1.025$ and 4.371 , respectively). Both theory and experiments give in the considered frequency range only a resonant peak at $\sigma / \sigma_{1}=1$, i.e., there is no resonant amplification at $\sigma_{2} / \sigma_{1}=1.52$. This means that the generalized coordinate $\beta_{-1}(t)$ describing the cross-flow at the screen dominates, while $\beta_{n}^{ \pm}, n \geq 1$ are of lower importance. Because $\beta_{n}^{ \pm}, n \geq 1$ determine the jump of the free surface at the screen, this jump does not provide a dominant contribution to the sloshing. The pressure loss coefficient $K$ plays the role of damping at the corresponding quadratic damping term in (25).

Figure 4 (panels a and b) shows that the numerical results by the analytical modal model generally agree with the experiments for the smaller nondimensional forcing amplitude $\eta_{2 a} / l \approx 0.001$. We see larger theoretical values relative to experiments in case (a) for $\mathrm{Sn}=0.328$. This fact can, in part, be related to non-accurate prediction of $K$ by empirical formulas (8). As we reported ahead of (8), the values of the empirical formula do not depend on the Reynolds number and assume $\mathrm{KC}>2$. An alternative is the table with empirical $K$ in [16, pp. 314-316]. The latter values do not depend on $\mathrm{KC}$ and the Reynolds number. We could have tried to fit the $K$-value to agree with the experimental results and, in that way, for instance, detect a KC-dependence. However, a procedure like that is questionable because it does not account for the fact that a reason for discrepancies can be due to nonlinear free-surface effects. In the presented calculations from Fig. $4 \mathrm{a}, K=1.025$ leads to $\zeta_{a} / \eta_{2 a}=50$ for $\sigma / \sigma_{1}=1$, but $K=1.32$ (this value of $K$ follows from the empirical loss coefficients in [16, p. 314]) gives $\zeta_{a} / \eta_{2 a}=43.5$. Because $\beta_{-1}$ dominates, one can, in a qualitative analysis, neglect the $\beta_{n}^{ \pm}$-quantities from (25) which, as explained previously, are responsible for the free-surface jump at the screen. This means that (25) becomes similar to a single-dimensional mass-spring system with quadratic damping where the damping coefficient is proportional to $K$ and, therefore, the screen should act as a damper. As shown in [25, Chap. 3] for a lightly damped one-degree of mass-spring system, the resonant nondimensional response $\zeta_{a} / \eta_{2 a}$ should then be proportional to $1 / \sqrt{K \eta_{2 a} / l}$. The nondimensional resonant response is consistent with this general prediction. 

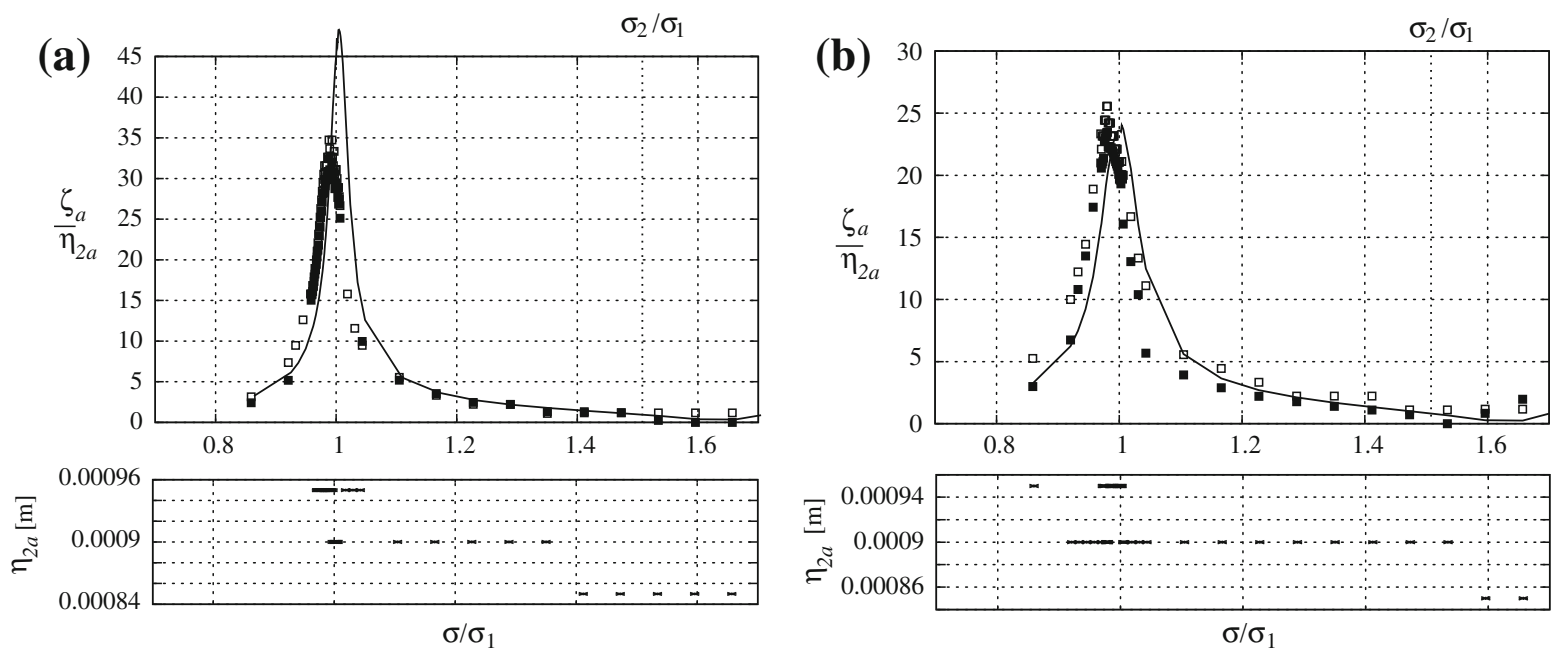

(c)

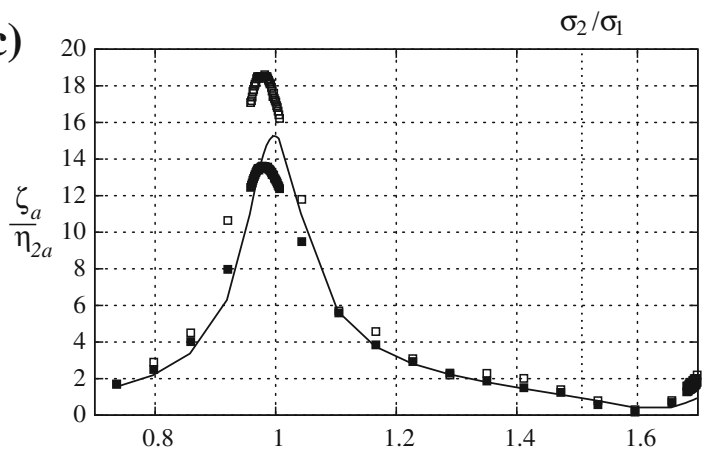

$\sigma / \sigma_{1}$
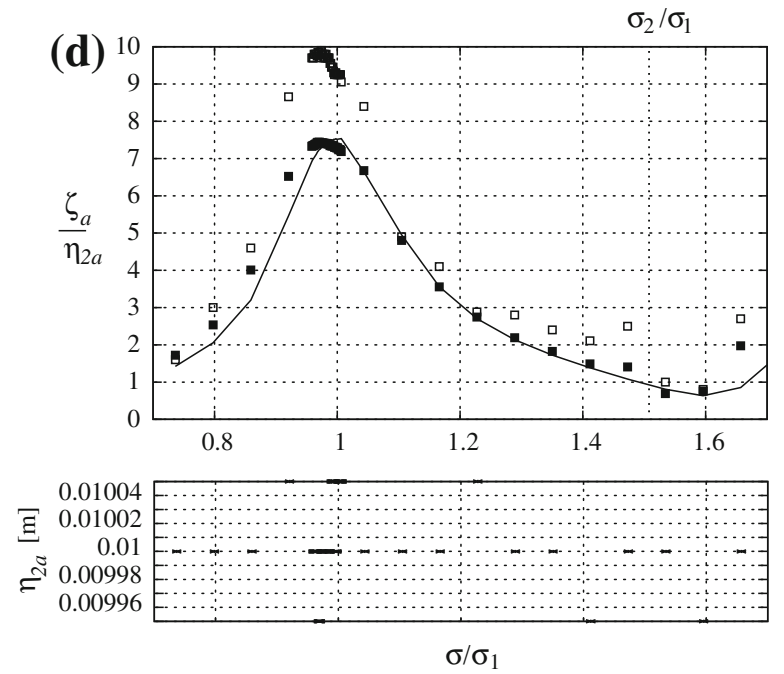

Fig. 4 Nondimensional theoretical (solid line) and experimental (filled and empty boxes) maximum wave elevations (denoted as $\zeta_{a}$ ) at the wave probe $1 \mathrm{~cm}$ away from the wall versus nondimensional forcing frequency $\sigma / \sigma_{1}$. The empty boxes represent the actual measured maximum wave elevations, but the solid (filled) boxes denote amplitude of the primary Fourier harmonics of the measured signal. Because the experimental horizontal forcing amplitude $\eta_{2 a}$ slightly varies with changing the forcing frequency, their dimensional values are also presented; this variation is taken into account in the numerical analysis. Panels $\mathbf{a}$ and $\mathbf{b}$ present the case of smaller forcing amplitudes $\left(\eta_{2 a} / l \approx 0.001\right)$, but $\mathbf{c}$ and $\mathbf{d}$ demonstrate the case of larger forcing amplitudes $\left(\eta_{2 a} / l \approx 0.01\right)$. The left panels a and $\mathbf{c}$, corresponds to $\mathrm{Sn}=0.328(K=1.025)$. The right panels, $\mathbf{b}$ and $\mathbf{d}$, show results for $\mathrm{Sn}=0.52(K=4.371)$

A major reason why the experimental and theoretical peak values and corresponding frequencies do not agree perfectly are believed to be due to free-surface nonlinearities. The latter is well known from the potential-flow sloshing in a smooth (clean) tank, when a linear sloshing theory based on potential flow wrongly predicts an infinite resonance response. Nonlinear resonant free-surface effects cause transfer of energy to other, higher modes than the primary excited, dominant mode and, thereby, limit the response of the primary excited mode at the lowest resonant frequency. Another consequence of nonlinear sloshing in a clean tank with $h / l>0.3368 \ldots$ is a drift of the peak response to a lower frequency than the lowest natural frequency $\sigma_{1}$. The latter effect is evident in the experimental results. If the dissipation (damping) due to cross-flow through the screen had been sufficiently high, i.e., larger than theoretically predicted in this case, the nonlinear free-surface effect would be negligible. 

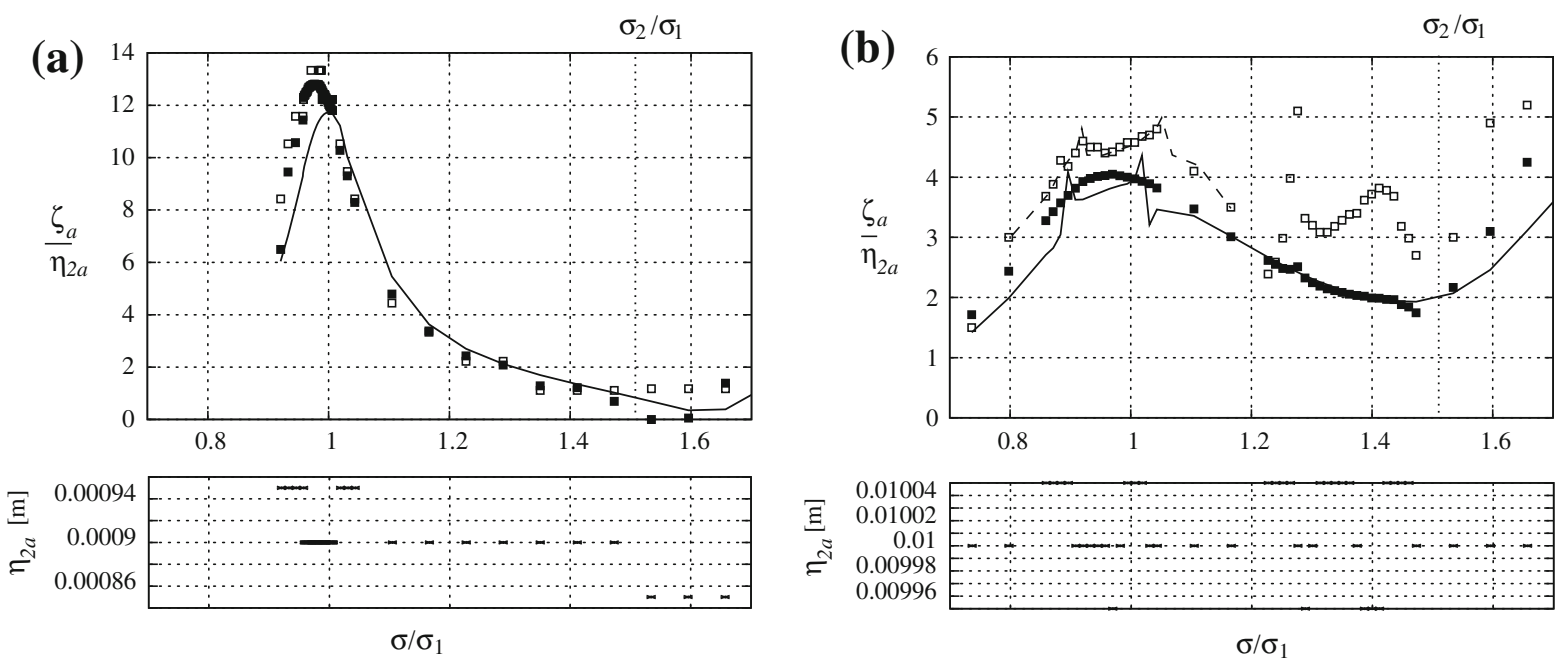

Fig. 5 Nondimensional theoretical (solid line) and experimental maximum wave elevations (denoted as $\zeta_{a}$ ) at the wave probe versus nondimensional forcing frequency $\sigma / \sigma_{1}$. The empty boxes represent the measured maximum wave elevations, but the solid boxes denote amplitudes of the primary harmonics, $\exp (\mathrm{i} \sigma t)$. The experimental variation of the forcing amplitudes is taken into account in the numerical analysis. $\mathrm{Sn}=0.713(K=19.89)$. Panel a corresponds to the lower excitation amplitudes, $\eta_{2 a} / l \approx 0.001$, and panel b implies $\eta_{2 a} / l \approx 0.01$

Fig. 6 The experimentally observed fallout of the liquid going through the screen into the air domain of the opposite compartment and subsequently impacting on the underlying free surface

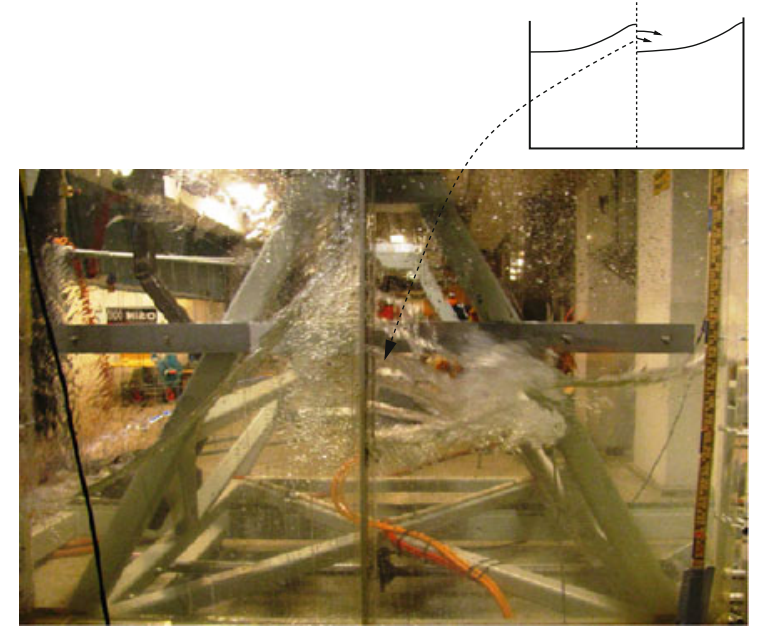

Furthermore, increasing the forcing amplitude may lead to a nonlinear amplification of higher modes which give a non-negligible contribution to the maximum steady-state wave elevation. This contribution is associated with higher harmonics. The cases (c) and (d) in Fig. 4 are for the larger nondimensional forcing amplitude $\eta_{2 a} / l \approx 0.01$. In these cases, the analytical modal model gives lower nondimensional values of the maximum steady-state elevation at the wave probes relative to the experimental measurements. To explain why this happens, we had to perform a Fourier analysis and extract the primary Fourier harmonics contribution from the measured output signal. It is marked by the filled boxes. Cases (a) and (b) show that the primary harmonics (associated with primary excited mode) clearly dominates for smaller forcing amplitude. This is not so for the larger forcing amplitude in cases (c) and (d), where higher harmonics (associated with higher modes) give sufficient contribution. Amplification of 

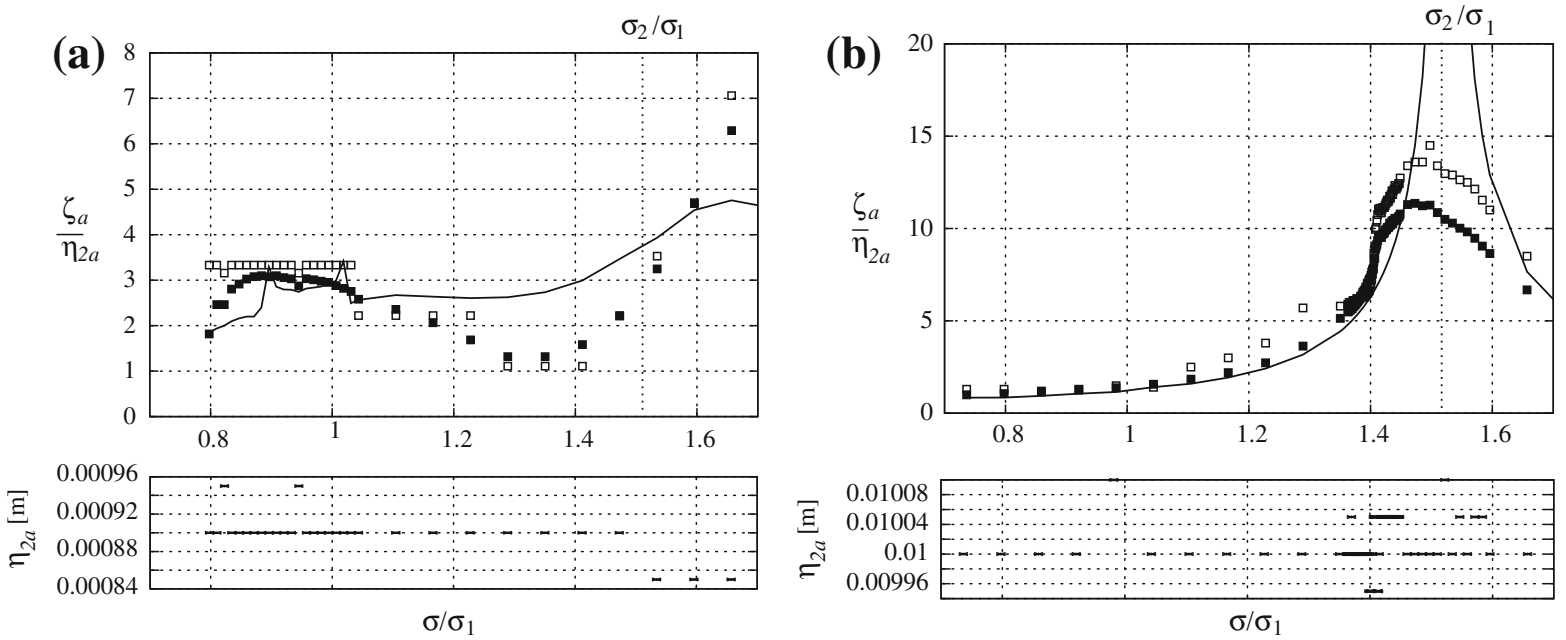

Fig. 7 The same as in Fig. 5, but for $\mathrm{Sn}=0.928(K=462.8)$. In case $\mathbf{b}$, the maximum peak at $\sigma / \sigma_{1}=\sigma_{2} / \sigma_{1}=1.52$ is computed to be $\zeta_{a} / \eta_{2 a}=50$
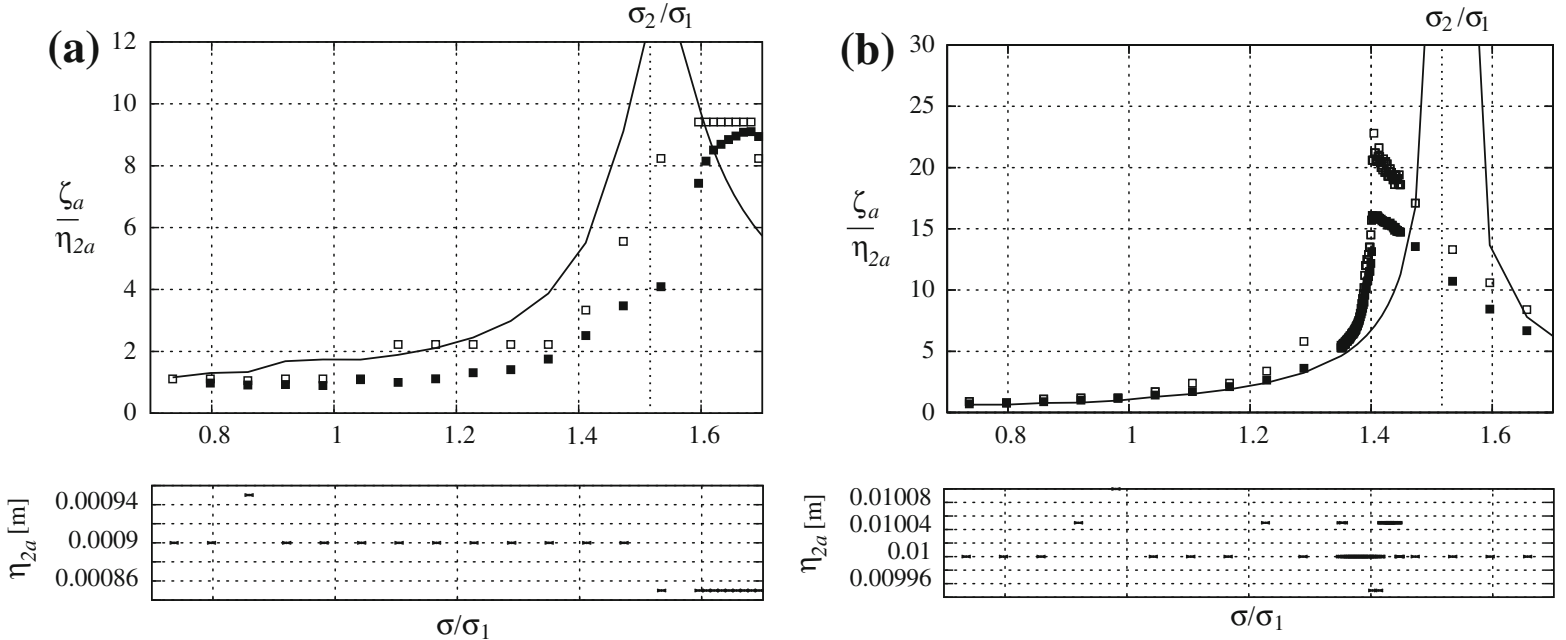

Fig. 8 The same as in Fig. 5, but for $\mathrm{Sn}=0.963(K=1,846)$. In case a, the maximum peak at $\sigma / \sigma_{1}=\sigma_{2} / \sigma_{1}=1.52$ is computed to be $\zeta_{a} / \eta_{2 a}=23$, but case $\mathbf{b}$ gives this theoretical maximum equal to 102

these harmonics with increasing forcing amplitude was extensively discussed in [27]. It was shown that this is a nonlinear free-surface effect. To describe this amplification, one should use the nonlinear multimodal theory which links the higher harmonics with nonlinear energy transfer from the lowest, primary excited mode to higher modes. Our theoretical model with linear free-surface conditions and damping due to the cross-flow through the screen demonstrates a good agreement with the primary harmonics contribution associated with the primary excited mode.

\subsubsection{Intermediate values of the solidity ratio}

Increasing the solidity ratios to $\mathrm{Sn}=0.713(K=19.89)$ leads to the theoretical and experimental nondimensional maximum steady-state wave elevations shown in Fig. 5. The generalized coordinate $\beta_{-1}(t)$ dominates with respect to $\beta_{n}^{ \pm}(t)$ in case (a), and, therefore, the system behaves as a one-degree lightly damped mass-spring system with quadratic damping. The consequence is that the resonant nondimensional response $\zeta_{a} / \eta_{2 a}$ is proportional to 
$1 / \sqrt{K \eta_{2 a} / l}$ which is consistent with the theoretical results in Fig. 4 for lower solidity ratios. The experimental results in case (a) show small influence of higher harmonics and there is good agreement with quasi-linear theory.

The larger excitation amplitude in Fig. $5 \mathrm{~b}$ leads to $\beta_{-1} \sim \beta_{n}^{ \pm}$with many dominant generalized coordinates. A consequence of coupling between modes is a double-peak response at the lowest natural sloshing frequency $\sigma_{1}$. The experimental primary harmonic response is, in general, in good agreement with the theoretical prediction. However, the experiments demonstrate the presence of non-negligible higher harmonics, for instance at $\sigma / \sigma_{1}=1.28$ and 1.4. A plausible reason is nonlinear free-surface effects. We do not know to what extent the water flow through the air illustrated in Fig. 6 matters. A water-jet flow that originates from the screen opening impacts on the underlying free surface and, thereby, can excite higher-harmonics wave (see discussion in [26]).

\subsubsection{Larger values of the solidity ratio}

Passage to higher solidity ratios is studied in Figs. 7 and 8 . Figure 7a corresponds to $\mathrm{Sn}=0.928$ and $\eta_{2 a} / l=0.001$. When $\sigma / \sigma_{1}$ is in the vicinity of $1, \beta_{-1} \sim \beta_{n}^{ \pm}$in the theoretical model. The consequence is that both cross-flow quadratic damping due to the screen and coupling between many generalized coordinates of the liquid motions matter. The result is a relatively small nondimensional wave amplitude response with the presence of a double peak. The agreement with the experiments is reasonable. The theoretical response is largest at $\sigma / \sigma_{1}=1.65$, i.e., at a higher frequency than the second natural sloshing frequency $\sigma_{2}=1.52 \sigma_{1}$. The experimental results do not show a clear effect of higher harmonics and agree that there is a peak response at $\sigma / \sigma_{1}=1.65$. However, the magnitudes of theoretical and experimental wave elevations differ at the second natural frequency with the experimental results being clearly higher. A reason can be errors in the theoretical damping model in the considered frequency range. Furthermore, there is a clear minimum in the experimental results at $\sigma / \sigma_{1}=1.35$ which is not supported by the theoretical model.

A very large theoretical amplification occurs at $\sigma / \sigma_{1}=1.52$ for the cases presented in Figs. $7 \mathrm{~b}$ and $8 \mathrm{a}, \mathrm{b}$. The reason is associated with the fact that $\beta_{n}^{ \pm} \gg \beta_{-1}$ in the theoretical model which can be explained as follows. Because there is a larger coefficient $K$ in the front of the quadratic damping term in (25), the generalized coordinate $\beta_{-1}$ is highly damped to become of non-dominant (higher order) with respect to $\beta_{n}^{ \pm}, n \geq 1$. The latter generalized coordinates are solutions of the linear equations (21) without damping terms and, therefore, the $\beta_{i}^{ \pm}$-related oscillations can only be reduced due to energy transfer to $\beta_{-1}$. The pressure-drop condition no longer plays the role of a damping mechanism, as it happened for lower and intermediate solidity ratios. An indication of the fact that the cross-flow at the screen does not act as a quadratic one-dimensional damping mechanism at $\sigma=\sigma_{2}$ is that we predict an increased nondimensional response $\zeta_{a} / \eta_{2 a}$ with increasing forcing amplitude $\eta_{2 a} / l$. The generalized coordinates $\beta_{n}^{ \pm}$governed by the linear non-damped linear oscillator equations (21) should determine both the primary response as well as the corresponding resonance peak which is now expected at $\sigma=\sigma_{2}$. The latter fact means that the screen can in a first approximation be considered as an unperforated wall which isolates wave motions in the compartments whose interplay is now associated with a higher-order flow component $\beta_{-1}$. It was shown in [6, Sect. 6.8] that increasing the excitation amplitude causes a decrease in the threshold value of the solidity ratio for which the screen changes its role from a damper to an isolator of the compartment tank. This explains why we see a clear resonance peak at $\sigma / \sigma_{1}=\sigma_{2} / \sigma_{1}=1.52$ and not at $\sigma / \sigma_{1}=1$ in Fig. 7 b. Case (a) in Fig. 7 with $\eta_{2 a} / l=0.001$ corresponds to a Sn-value where the dominant resonant response changes from being at $\sigma / \sigma_{1}=1$ to $\sigma / \sigma_{1}=1.52$.

The experimental results in a frequency range around the second natural frequency do not agree well with the theoretical results in Figs. 7b and 8a, b. The experiments show a frequency of the peak response that is lower than $\sigma_{2}$ for $\eta_{2 a} / l=0.01$ (Figs. $7 \mathrm{~b}, 8 \mathrm{~b}$ ) and larger than $\sigma_{2}$ for $\eta_{2 a} / l=0.001$ (Fig. 8a). Furthermore, the maximum experimental values are clearly lower than the theoretical values. The presence of higher-harmonics contribution in the experiments indicates nonlinear free-surface effects causing transfer of energy from lowest primary excited mode to higher modes. The experimental behavior for $\eta_{2 a} / l=0.01 l$ is consistent with a theoretical nonlinear behavior of resonant sloshing for depths that are larger than the critical depth in a rectangular tank with small damping. For instance, jumps between stable steady-state solution branches occur according to the multimodal method with nonlinear free-surface nonlinearity effects described in [6, Chap. 8]. The response has similarities 
with the response of a Duffing-type nonlinear oscillator. Because there is a small flow exchange between the tank compartments, the relevant theoretical tanks are the compartment tanks. We are not able to theoretically explain why the maximum experimental response occurs for a frequency larger than the second natural frequency in Fig. 8a with $\eta_{2 a} / l=0.001$ and $\mathrm{Sn}=0.963$.

\section{Concluding remarks}

A modal model based on linear free-surface conditions has been derived to describe sloshing in a two-dimensional rectangular tank with a slat-type screen in the middle. An empirical pressure-drop condition as well as continuity of the transverse velocity were used as transmission conditions between the two compartments. The derived modal model consists of two modal systems to describe sloshing in the two compartments, and an ordinary differential equation governing the generalized coordinate $\beta_{-1}(t)$ responsible for liquid flow between compartments. The first modal systems are similar to that in [6, Chap. 5], but with other right-hand sides. These right-hand sides include terms depending on $\beta_{-1}(t)$. The last differential equation with respect to $\beta_{-1}(t)$ contains the nonlinear, $\dot{\beta}_{-1}\left|\dot{\beta}_{-1}\right|-$ term coming from the pressure-drop condition.

The theoretical pressure-drop condition across the screen plays the role of a damper for low and intermediate solidity ratios. For low solidity ratio, this role is similar to that in a single-degree mass-spring system with quadratic damping. A consequence of the latter fact is that the resonant nondimensional response $\zeta_{a} / \eta_{2 a}$ is proportional to $1 / \sqrt{K \eta_{2 a} / l}$ where $K$ is the pressure-drop coefficient. The same similarity to a spring-mass system is applicable for intermediate solidity ratios and smaller forcing amplitudes $\left(\eta_{2 a} / l=0.001\right.$ in our studies). However, increasing the forcing amplitude makes inapplicable the single-degree mechanical analogy. There are then many generalized coordinates and corresponding natural modes which should give comparable dominant contributions, and the actual response results from a complex interplay between them. The pressure-drop condition does not act as a damping mechanism for higher solidity ratios, but causes the lowest resonance sloshing frequency to increase to the lowest sloshing frequency in the tank compartments isolated by the screen as an unperforated wall. Which lower solidity ratio causes the change in the resonance frequency under harmonic sway excitation in a two-dimensional tank depends on the liquid depth-to-the-tank-width ratio and the ratio between the sway amplitude and the tank width.

Even though we use linear free-surface conditions and a screen-averaged pressure-drop condition, comparison of the numerical results by the derived analytical modal model and new experimental model tests on steady-state wave elevations show satisfactory agreement in many cases. The most serious discrepancy can be explained by the free-surface nonlinearity. Mathematically, the nonlinearity at the second natural frequency $\sigma_{2}$ (for larger solidity ratios) can be handled by replacing the left-hand sides of (21) with the corresponding adaptive modal system taken from paper [27]. However, we anticipate a non-trivial analysis accounting for a very special liquid flow associated with a liquid jet that originates at the screen, goes through the air and subsequently impacts on the underlying free surface (see Fig. 6).

Because of the specific slat-type screen, we adopted a mean (screen-averaged) pressure-drop condition. Generally speaking, the sloshing problem needs a modification of this condition that accounts for the local approach velocity and pressure drop at each opening in the screen.

Open Access This article is distributed under the terms of the Creative Commons Attribution Noncommercial License which permits any noncommercial use, distribution, and reproduction in any medium, provided the original author(s) and source are credited.

\section{References}

1. Abramson H (1966) The dynamic behavior of liquids in moving containers. Washington DC, NASA SP-106

2. Warnitchai P, Pinkaew T (1998) Modelling of liquid sloshing in rectangular tanks with flow-dampening devices. Eng Struct 20:593600

3. Tait MJ, El Damatty AA, Isyumov N, Siddique MR (2005) Numerical flow models to simulate tuned liquid dampers (TLD) with slat screens. J Fluids Struct 20:1007-1023

4. Tait MJ (2008) Modelling and preliminary design of a structure-TLD system. Eng Struct 30:2644-2655 
5. Maravani M, Hamed MS (in press) Numerical modeling of sloshing in a turned liquid damper outfitted with a submerged slat screen. Int J Numer Methods Fluids. doi:10.1002/fld.2250

6. Faltinsen OM, Timokha AN (2009) Sloshing. Cambridge University Press, New York

7. Kaneko S, Ishikawa M (1992) Modelling of tuned liquid damper with submerged nets. ASME PVP 247:185-203

8. Kaneko S, Ishikawa M (1999) Modelling of tuned liquid damper with submerged nets. Trans ASME, J Press Vessel Technol 121:334-341

9. Kaneko S, Mizota Y (2000) Dynamical modelling of deepwater-type cylindrical tuned liquid damper with a submerged net. Trans ASME, J Press Vessel Technol 122:96-104

10. Tuck EO (1971) Transmission of water waves through small apertures. J Fluid Mech 49:65-74

11. Tuck EO (1975) Matching problems involving flow through small holes. Adv Appl Mech 15:89-157

12. Evans DV (1990) The use of porous screens as wave dampers in narrow wave tanks. J Eng Math 24:203-212

13. Chwang AT, Chan AT (1998) Interaction between porous media and wave motion. Annu Rev Fluid Mech 30:53-84

14. Straughan B (2008) Stability and wave motion in porous media. Springer Verlag, Berlin

15. Laws EM, Livesey JL (1978) Flow through screens. Annu Rev Fluid Mech 10:247-266

16. Blevins RD (1992) Applied fluid dynamics. Krieger Publishing Company, Malabar, FL

17. Molin B (2001) On the added mass and damping of periodic arrays of fully or partially porous disks. J Fluid Struct 15:275-290

18. Kimmoun O, Molin B, Moubayed W (2001) Second-order analysis of the interaction of a regular wave train with a vertical perforated wall. In: Proceedings of 8 èmes Journèes de l'Hydrodynamique, 5, 6, 7 March 2001, Nantes

19. Baines WD, Peterson EG (1951) An investigation of flow though screens. ASME Trans 73:467-479

20. Weisbach J (1855) Die Experimental-Hydraulik. Engelehardt, Freiburg

21. Faltinsen OM (1974) Nonlinear theory of sloshing in rectangular tanks. J Ship Res 18:224-241

22. Faltinsen OM, Rognebakke OF, Lukovsky IA, Timokha AN (2000) Multidimensional modal analysis of nonlinear sloshing in a rectangular tank with finite water depth. J Fluid Mech 407:201-234

23. Agranovich MS (1996) Elliptic boundary problems. In: Encyclopedia of mathematical sciences, vol 79. Springer, Berlin, pp 1-146

24. Karlin S, Studden WJ (1966) Tschebyscheff systems with applications in analysis and statistics. Wiley, New York

25. Faltinsen OM (1990) Sea loads on ships and offshore structures. Cambridge University Press, New York

26. Faltinsen OM, Rognebakke OF, Timokha AN (2006) Transient and steady-state amplitudes of resonant three-dimensional sloshing in a square base tank with a finite fluid depth. Phys Fluids 18, Art. No. 012103:1-14

27. Faltinsen OM, Timokha AN (2001) Adaptive multimodal approach to nonlinear sloshing in a rectangular tank. J Fluid Mech 432:167-200 